\title{
Nuclear disconnection within the amygdala reveals a direct pathway to fear
}

\author{
Stephanie A. Jimenez ${ }^{1}$ and Stephen Maren ${ }^{1,2,3}$ \\ ${ }^{1}$ Neuroscience Program, University of Michigan, Ann Arbor, Michigan 48109-1043, USA; ${ }^{2}$ Department of Psychology, University of \\ Michigan, Ann Arbor, Michigan 48109-1043, USA
}

\begin{abstract}
It is widely believed that a descending serial circuit consisting of neural projections from the basolateral complex (BLA) to the central nucleus (CEA) of the amygdala mediates fear expression. Here we directly test this hypothesis and show that disconnecting the BLA and CEA with asymmetric neurotoxic lesions after Pavlovian fear conditioning in rats completely abolishes the expression of conditional freezing. These results demonstrate that neural projections from the BLA to CEA are essential for the expression of learned fear responses.
\end{abstract}

[Supplemental material is available online at http:// www.learnmem.org.]

Long-standing anatomical models of the brain circuitry underlying learned fear posit serial flow of information through the amygdala to engage the expression of fear responses (LeDoux 2000; Maren 2001). Specifically, conditioning-induced plasticity in the lateral nucleus of the amygdala (LA) is thought to drive learned fear through excitatory axonal projections to the basal nuclei of the amygdala (BA; basolateral and basomedial nuclei), which in turn send unidirectional and excitatory synaptic projections to the medial division of the central nucleus of the amygdala (CEm). Neurons in the CEm project to brain structures involved in the production of a variety of fear responses (Pitkänen et al. 1997; Swanson and Petrovich 1998). Alternatively, neurons in LA might excite neurons in CEm by limiting inhibitory input from the intercalated cell masses (ITC) interposed between the basolateral complex (BLA; lateral and basal nuclei) and the central nucleus of the amygdala (CEA) (Paré et al. 2004). In either case, the BLA is well positioned to drive learned fear responses via anatomical connections with the CEA.

Although an extensive literature demonstrates the importance of both the BLA and CEA in the acquisition and expression of fear (LeDoux et al. 1990; Lee et al. 1996; Amorapanth et al. 2000; Goosens and Maren 2001), it is not known whether a functional connection between the two structures is essential for the expression of learned fear. Indeed, the BLA and CEA make independent contributions to aversively motivated learning under some conditions (Killcross et al. 1997; Amorapanth et al. 2000). Moreover, recent work in appetitive conditioning paradigms challenges the necessity of serial circuits in the amygdala for associative learning processes (Holland and Gallagher 1999; Everitt et al. 2003; Balleine and Killcross 2006). It is therefore essential to determine whether serial connections between the BLA and CEA are involved in the expression of fear memories as widely assumed in the literature.

To address this issue, we made asymmetric neurotoxic lesions of the BLA and CEA after Pavlovian fear conditioning in rats. That is, we placed BLA lesions in one hemisphere and CEA lesions in the contralateral hemisphere, thereby producing a functional disconnection of the two brain regions. Control animals received neurotoxic BLA and CEA lesions in the same hemisphere, thereby

\footnotetext{
${ }^{3}$ Corresponding author.
}

E-mail maren@umich.edu; fax (734) 763-7480.

Article is online at http://www.learnmem.org/cgi/doi/10.1101/lm.1607109. leaving both structures and their connections intact in one hemisphere. This disconnection strategy (e.g., Olton et al. 1982) capitalizes on the fact that projections from the BLA to CEA are both ipsilateral and unidirectional (Pitkänen et al. 1997). It has been used by several groups to assess the contribution of connections between brain areas to learning and memory processes, including conditioned stimulus processing (Han et al. 1999) and appetitive spatial learning (Ito et al. 2008), for example.

Fear conditioning was conducted in standard observation chambers (see Supplemental Methods) and consisted of five pairings of an auditory conditional stimulus (CS) $(2 \mathrm{kHz}, 10 \mathrm{sec}$, $80 \mathrm{~dB}$ ) with a footshock unconditioned stimulus (US) (2 sec, 1 $\mathrm{mA}$ ); the intertrial interval (ITI) was $1 \mathrm{~min}$. Freezing behavior served as the measure of conditional fear. Twenty-four hours after fear conditioning, the rats were deeply anesthetized, and amygdala lesions were made by infusing $N$-methyl-D-aspartate (NMDA; $20 \mathrm{mg} / \mathrm{mL}$ in $100 \mathrm{mM}$ phosphate buffered saline with a $\mathrm{pH}$ 7.4; Sigma) into the CEA and BLA through a 28-g injection cannula attached to a Hamilton syringe via polyethylene tubing. Control rats received unilateral lesions of the BLA and CEA in the same hemisphere (IPSI, $n=13$ ) or sham surgery (SHAM, $n=16$ ), whereas experimental rats received the unilateral BLA and CEA lesions in opposite hemispheres (CONTRA, $n=11$ ). Hence, functional connectivity between the BLA and CEA was left intact in one hemisphere among rats in the IPSI group, whereas the asymmetric lesions in rats in the CONTRA group eliminated this connection. Retention tests assessing fear to the conditioning context and tone CS were conducted in separate sessions one week after recovery from surgery. Histological examination of coronal brain sections obtained from the subjects after the experiment revealed selective CEA and BLA lesions in each group; representative lesions are illustrated in Figure 1, A and B. The lesions spared fibers of passage (Fig. 1C).

As shown in Figure 1D (left panel), all rats acquired similar levels of conditional freezing (collapsed across the CS and ITI for each trial) by the end of the presurgical fear conditioning session (main effect of trial, $F_{(5,185)}=42.1, P<0.0001$ ); there was neither a main effect of group $(F<1)$ nor a group $\times$ trial interaction $(F<$ 1.3). One week after recovery from surgery, conditional freezing to the conditioning context and the auditory CS was assessed in separate retention tests. Importantly, functional disconnection of the BLA and the CEA after fear conditioning severely impaired the expression of conditioned freezing to both the conditioning 
A

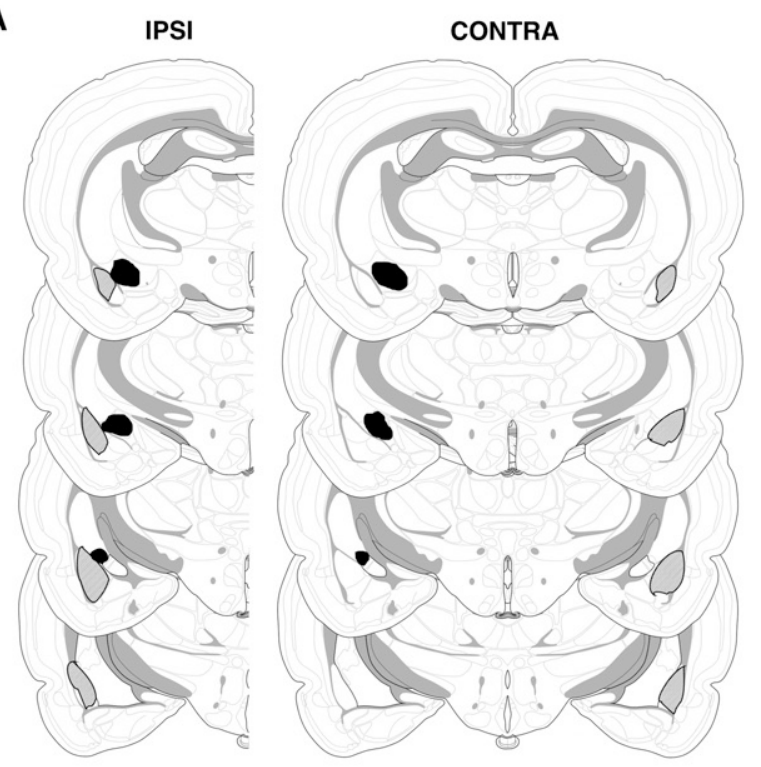

B

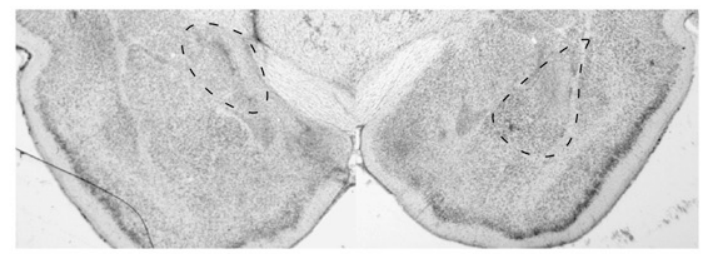

C

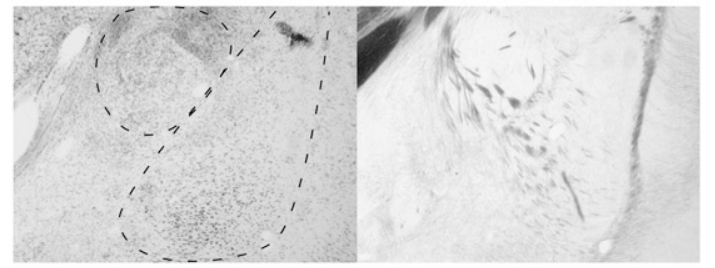

D

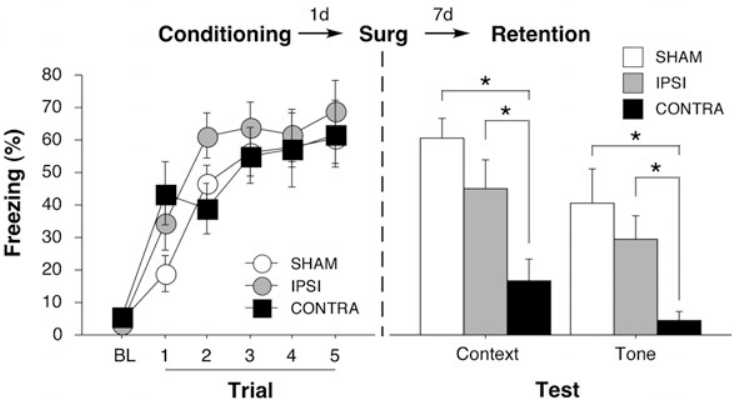

Figure 1. Disconnection of the BLA and CEA impairs the expression of conditional fear. $(A)$ Schematic representation of a typical BLA (shaded) and CEA (black) lesions among rats with unilateral lesions placed in the same hemisphere (IPSI) or in opposite hemispheres (CONTRA). (B) Photomicrograph of a thionin-stained coronal section from a representative rat in the CONTRA group. The image has been cropped to include only the left and right amygdale. Broken lines encircle CEA and BLA lesions in the left and right hemispheres, respectively. (C, left) Photomicrograph of a thioninstained coronal section from a representative rat in the IPSI group. The image has been cropped to focus on the lesion in the right amygdala. Broken lines encircle the CEA and BLA lesions. (C, right) Photomicrograph of an $\mathrm{AuCl}$-stained coronal section adjacent to that shown at left indicates that NMDA infusions into the amygdala did not damage myelinated axons in the vicinity of the lesion. (D) Mean percentage of freezing ( \pm SEM) during the conditioning session (collapsed across CSs and ITIs) and retention tests in rats in each of the three groups. The expression of fear to the conditioning context and tone CS was severely impaired by disconnection of the BLA and CEA in the CONTRA group. ${ }^{*} P<0.05$. context and the auditory CS (Fig. 1D, right). Indeed, the expression of fear to the auditory CS was essentially abolished. Freezing among rats in the CONTRA group was significantly lower than that in both the SHAM and IPSI groups during both retention tests (main effect of group, $F_{(2,37)}=9.98, P<0.001$ ), and this difference did not interact with the nature of the retention test $(F<1)$. Hence, functional disconnection of the BLA and CEA produced an impairment in the expression of conditional fear that was much greater than that produced by comparable lesions that spared this connection.

The logic of the disconnection procedure requires that focal CEA and BLA lesions in each hemisphere do not damage the adjacent BLA or CEA, respectively. For instance, if CEA lesions produce retrograde degeneration in the neighboring BLA, rats in the CONTRA group would effectively have bilateral lesions in the BLA. Such an outcome would be expected to yield the massive deficits in conditional freezing that we have observed. Inspection of thionin-stained coronal sections suggested that, as in previous studies (Goosens and Maren 2001), our lesions were indeed selective for the targeted areas (Fig. 1B). However, to increase our confidence that the BLA adjacent to a CEA lesion was in fact functional, we performed c-fos immunohistochemistry on brain sections obtained from a separate group of rats trained and tested as previously described (SHAM, CONTRA, and IPSI groups; $n=8$ per group). In addition to these rats, we included a group of rats that were not conditioned (NO-SHOCK, $n=8$ ) to quantify fearinduced increases in c-fos expression. All rats were sacrificed 90 min after the retention test to the auditory CS. Amygdaloid c-fos expression in the SHAM rats and the intact hemisphere of the IPSI rats did not differ, and these groups were therefore collapsed into a single SHAM group.

As shown in Figure 2, SHAM rats exhibited a greater density of c-fos positive nuclei in both the CEA and BLA relative to nonshocked controls. Quantification of these data confirmed this observation and revealed significant differences in c-fos expression among the groups in both the CEA (Fig. 2, left; $F_{(2,40)}=5.59$, $P<0.01)$ and the BLA (Fig. 2, right; $F_{(2,40)}=5.07, P<0.01$ ). Importantly, BLA c-fos expression adjacent to CEA lesions in the CONTRA group was similar to that in intact SHAM rats, and significantly greater than that in nonshocked controls (Fig. 2B, right). This suggests that the failure of CONTRA rats to express conditional fear responses was not due to a failure to engage the intact BLA but rather to the functional disconnection of BLA activity from CEA output. Indeed, the CEA adjacent to a BLA lesion exhibited a marked reduction in c-fos expression relative to SHAM controls (Fig. 2, left), indicating that BLA lesions failed to drive ipsilateral CEA neurons important for the expression of learned fear. It is possible that the absence of c-fos expression in the CEA, in this case, is due to encroachment of the adjacent BLA lesion. However, thionin-stained sections revealed that BLA lesions were selective. Moreover, we observed intact BLA c-fos expression in rats with adjacent CEA lesions suggesting that nearby lesions per se do not disrupt c-fos expression.

These results reveal that a functional connection between the BLA and CEA is required for the expression of learned fear. Considering that BLA projections to the CEA are largely unidirectional, our data reveal that a serial circuit from the BLA to the CEA mediates the expression of conditional fear responses. Consistent with this view, there are numerous reports that permanent lesions or reversible inactivation of either the BLA or CEA prevent the expression of conditioned fear (Lee et al. 1996; Maren 1999; Zimmerman et al. 2007). It is now apparent that the necessity for both the BLA and CEA in fear expression arises from the functional connectivity between them. Anatomically, this connection might involve a direct excitatory projection from BA to 


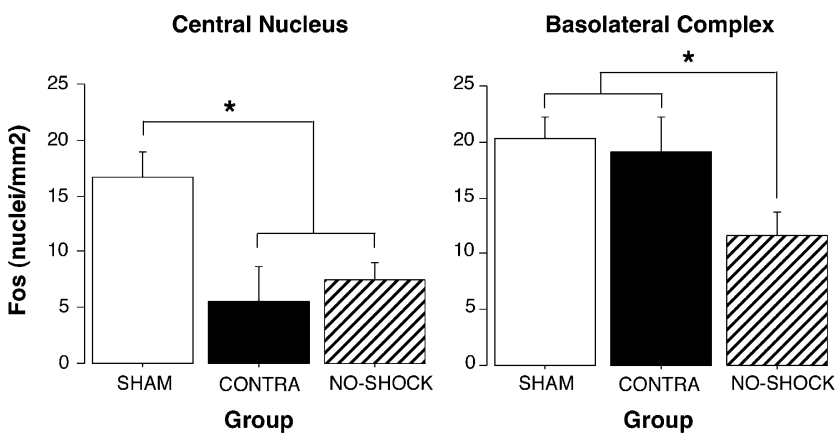

Figure 2. Functional disconnection of the BLA and CEA revealed by c-fos expression. Mean density ( \pm SEM) of c-fos positive nuclei in the CEA (left) and BLA (right) in rats from each of the three groups. Disconnection of the BLA and CEA eliminated fear-related increases in c-fos expression in the CEA (left), but not the BLA (right). ${ }^{*} P<0.05$.

CEm (Paré et al. 1999) or indirect projections from LA to ITC and the lateral division of the central nucleus (CEl), both of which project to CEm (Smith and Paré 1994; Paré et al. 2004). Recent data reveal, however, that selective immunotoxic lesions of the ITC do not impair the expression of conditioned freezing (Likhtik et al. 2008). Moreover, CEl projections to CEm are inhibitory, making it unlikely that that an LA-CEl projection drives learned fear responses via CEm (Paré et al. 2004). Hence, it appears that the most likely route by which fear CSs drive learned fear involves projections from BA to CEm. Consistent with this, selective BA lesions disrupt the expression of conditioned freezing when made either before (Goosens and Maren 2001) or after (Anglada-Figueroa and Quirk 2005) fear conditioning.

The dependence of conditional fear on a serial circuit between the BLA and CEA stands in contrast to the independent roles these areas have been proposed to play in appetitive conditioning paradigms (Holland and Gallagher 1999; Everitt et al. 2003; Balleine and Killcross 2006). For example, CEA, but not BLA, lesions have been reported to produce deficits in the acquisition and expression of autoshaped conditioned responses (CRs), indicating that the CEA has an independent contribution to CR expression for food-motivated responses (Parkinson et al. 2000). Moreover, the BLA has a role in the attribution of incentive salience to rewarding stimuli independent of the generation of CRs to those stimuli (Hatfield et al. 1996). However, in aversive conditioning, it appears that the BLA may not encode the motivational properties of the shock US (Rabinak and Maren 2008), but rather CS-US associations that are essential for organizing conditional fear responses by the CEA. Indeed, there is an emerging body of data suggesting that these associations may be established not only in sensory afferents in the BLA but also in the CEA (Wilensky et al. 2006; Zimmerman et al. 2007). Synaptic plasticity in projections from BA to CEm may be the essential substrate underlying the functional connectivity between these structures that is essential for the expression of fear memory.

\section{Acknowledgments}

We thank Dr. Ewelina Knapska for assistance with immunohistochemistry, Caitlin Orsini for assistance with behavioral testing, and Joshua Zimmerman for commenting on an earlier draft of the manuscript. The research reported in this paper was supported by a National Institute of Health grant to S.M. (R01MH073655).

\section{References}

Anglada-Figueroa D, Quirk GJ. 2005. Lesions of the basal amygdala block expression of conditioned fear but not extinction. J Neuosci 25: 96809685 .

Amorapanth P, Ledoux JE, Nader K. 2000. Different lateral amygdala outputs mediate reactions and actions elicited by a fear-arousing stimulus. Nat Neurosci 3: 74-79.

Balleine BW, Killcross S. 2006. Parallel incentive processing: An integrated view of amygdala function. Trends Neurosci 29: 272-279.

Everitt BJ, Cardinal RN, Parkinson JA, Robbins TW. 2003. Appetitive behavior: Impact of amygdala-dependent mechanisms of emotional learning. Ann N Y Acad Sci 985: 233-250.

Goosens KA, Maren S. 2001. Contextual and auditory fear conditioning are mediated by the lateral, basal, and central amygdaloid nuclei in rats. Learn Mem 8: 148-155.

Han JS, Holland PC, Gallagher M. 1999. Disconnection of the amygdala central nucleus and substantia innominata/nucleus basalis disrupts increments in conditioned stimulus processing in rats. Behav Neurosci 13: $143-151$.

Hatfield T, Han JS, Conley M, Gallagher M, Holland P. 1996. Neurotoxic lesions of basolateral, but not central, amygdala interfere with Pavlovian second-order conditioning and reinforcer devaluation effects. J Neurosci 16: 5256-5265.

Holland PC, Gallagher M. 1999. Amygdala circuitry in attentional and representational processes. Trends Cogn Sci 3: 65-73.

Ito R, Robbins TW, Pennartz CM, Everitt BJ. 2008. Functional interaction between the hippocampus and nucleus accumbens shell is necessary for the acquisition of appetitive spatial context conditioning. J Neurosci 28: 6950-6959.

Killcross S, Robbins TW, Everitt BJ. 1997. Different types of fear-conditioned behaviour mediated by separate nuclei within the amygdala. Nature 388: $377-380$.

LeDoux JE. 2000. Emotion circuits in the brain. Annu Rev Neurosci 23: 155184.

LeDoux JE, Cicchetti P, Xagoraris A, Romanski LM. 1990. The lateral amygdaloid nucleus: Sensory interface of the amygdala in fear conditioning. J Neurosci 10: 1062-1069.

Lee H, Walker D, Davis M. 1996. Lack of a temporal gradient of retrograde amnesia following NMDA-induced lesions of the basolateral amygdala assessed with the fear-potentiated startle paradigm. Behav Neurosci 110: 836-869.

Likhtik E, Popa D, Aspergis-Schoute J, Fidarco GA, Paré D. 2008. Amygdala intercalated neurons are required for expression of fear extinction. Nature 454: 642-645.

Maren S. 1999. Neurotoxic basolateral amygdala lesions impair learning and memory but not the performance of conditional fear in rats. $J$ Neurosci 19: 8696-8703.

Maren S. 2001. Neurobiology of Pavlovian fear conditioning. Annu Rev Neurosci 24: 897-931.

Olton DS, Walker JA, Wolf WA. 1982. A disconnection analysis of hippocampal function. Brain Res 233: 241-253.

Paré D, Smith Y, Paré JF. 1999. Intra-amygdaloid projections of the basolateral and basomedial nuclei in the cat: Phaseolus vulgarisleucoagglutinin anterograde tracing at the light and electron microscopic level. Neuroscience 69: 567-583.

Paré D, Quirk GJ, Ledoux JE. 2004. New vistas on amygdala networks in conditioned fear. J Neurophysiol 92: 1-9.

Parkinson JA, Robbins TW, Everitt BJ. 2000. Dissociable roles of the central and basolateral amygdala in appetitive emotional learning. Eur $J$ Neurosci 12: 405-413.

Pitkänen A, Savander V, LeDoux JE. 1997. Organization of intra-amygdaloid circuitries in the rat: An emerging framework for understanding functions of the amygdala. Trends Neurosci 20: 517-523.

Rabinak CA, Maren S. 2008. Associative structure of fear memory after basolateral amygdala lesions in rats. Behav Neurosci 122: 1284-1294.

Smith Y, Paré D. 1994. Intra-amygdaloid projections of the lateral nucleus in the cast: PHA-L anterograde labeling combined with post-embedding GABA and glutamate immunohistochemistry. J Comp Neurol 342: 232-248.

Swanson LW, Petrovich GD. 1998. What is the amygdala? Trends Neurosci 21: $323-331$.

Wilensky AE, Glenn ES, Kristensen MP, Ledoux JE. 2006. Rethinking the fear circuit: The central nucleus of the amygdala is required for the acquisition, consolidation, and expression of Pavlovian fear conditioning. J Neurosci 26: 12387-12396.

Zimmerman JZ, Rabinak CA, McLachlan IG, Maren S. 2007. The central nucleus of the amygdala is essential for acquiring and expressing conditional fear after overtraining. Learn Mem 9: 634-644.

Received August 25, 2009; accepted in revised form October 12, 2009. 


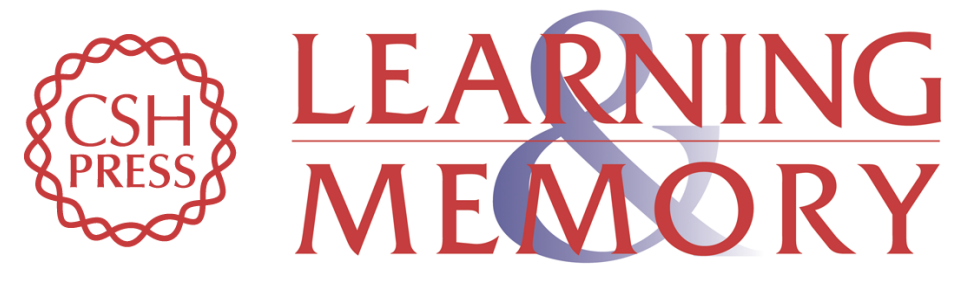

\section{Nuclear disconnection within the amygdala reveals a direct pathway to fear}

Stephanie A. Jimenez and Stephen Maren

Learn. Mem. 2009, 16:

Access the most recent version at doi:10.1101//m.1607109

Supplemental http://learnmem.cshlp.org/content/suppl/2009/11/30/16.12.766.DC1
Material

References This article cites 26 articles, 6 of which can be accessed free at: http://learnmem.cshlp.org/content/16/12/766.full.html\#ref-list-1

License

Email Alerting Receive free email alerts when new articles cite this article - sign up in the box at the Service top right corner of the article or click here. 